\title{
Photoallergic contact dermatitis from benzydamine presenting mainly as lip dermatitis
}

\author{
Maria Miguel Canelas, José Carlos Cardoso, Margarida Gonçalo and Américo Figueiredo \\ Serviço de Dermatologia, Hospitais da Universidade de Coimbra, Praceta Mota Pinto, 3000-075 Coimbra, Portugal
} Background: Benzydamine, a non-steroidal anti-inflammatory drug (NSAID) in use for more than four
decades, has been reported to cause photosensitivity.

Objectives: To study the results of photopatch testing to benzydamine and the clinical features of the dermatitis during a 3-year period (2006-2008).

Patients and methods: During this period, 74 patients with photodermatoses were photopatch tested with an extended baseline series of allergens including benzydamine and in suspicious cases, with drugs that contain it. Test sites were irradiated on D2 with $5 \mathrm{~J} / \mathrm{cm}^{2}$ and readings were performed on D2 and D4.

Results: Ten patients (six females/four males), aged 21-84 years (mean 64.9) had a positive photopatch test to benzydamine $\left[1-5 \%\right.$ petrolatum (pet.) from Bial-Aristegui $\left.{ }^{\circledR}\right]$ and to drugs that contain it (Tantum verde ${ }^{\circledR}$ oral solution and Momen ${ }^{\circledR}$ gel). Nine patients had lower lip cheilitis and one lichenified eczema on photo-exposed sites.

Conclusion: Photosensitivity from both topical and systemic benzydamine has been occasionally described, mainly in southern Spain. Despite its widespread use and its known photosensitizing capacity, photoallergic contact dermatitis from benzydamine is probably underdiagnosed as the clinical presentation of mainly the lip and chin is not typical of photoallergic contact dermatitis and benzydamine is not part of most photoallergen series.

Key words: benzydamine; cheilitis; non-steroidal anti-inflammatory drug; photoallergic contact dermatitis. (C) John Wiley \& Sons A/S, 2010.

Conflict of interests: The authors have declared no conflicts.

Accepted for publication 13 January 2010

Benzydamine (CAS number 642-72-8) is an indazole non-steroidal anti-inflammatory drug (NSAID) that has analgesic, anti-inflammatory, and antipyretic properties and has been widely used across Europe for nearly four decades (1). Benzydamine is available as a mouth wash for the relief of sore throats, as an antiseptic vagina douche, and as an anti-inflammatory cream and gel to treat soft tissues (1-3). Recently, benzydamine was formulated in a muco-adhesive gel designed for oral ulcers and for radiation-induced mucositis of the oral, rectal, or vaginal mucosa in cancer treatment $(4,5)$.

Photoallergic contact dermatitis from topical and systemic benzydamine is known $(1-3,6,7)$. Since
July 2006 after diagnosing a case of cheilitis as a manifestation of photoallergic contact dermatitis from benzydamine, this NSAID has been included in our photoallergen patch test series and nine additional cases of photoallergic contact dermatitis have been diagnosed.

\section{Patients and Methods}

During a 3-year period (2006-2008), 74 patients (53 females/21 males) with photodermatoses or facial dermatitis with aggravation following sun exposure were patch tested with an extended baseline series and photopatch tested with a photoallergen series 


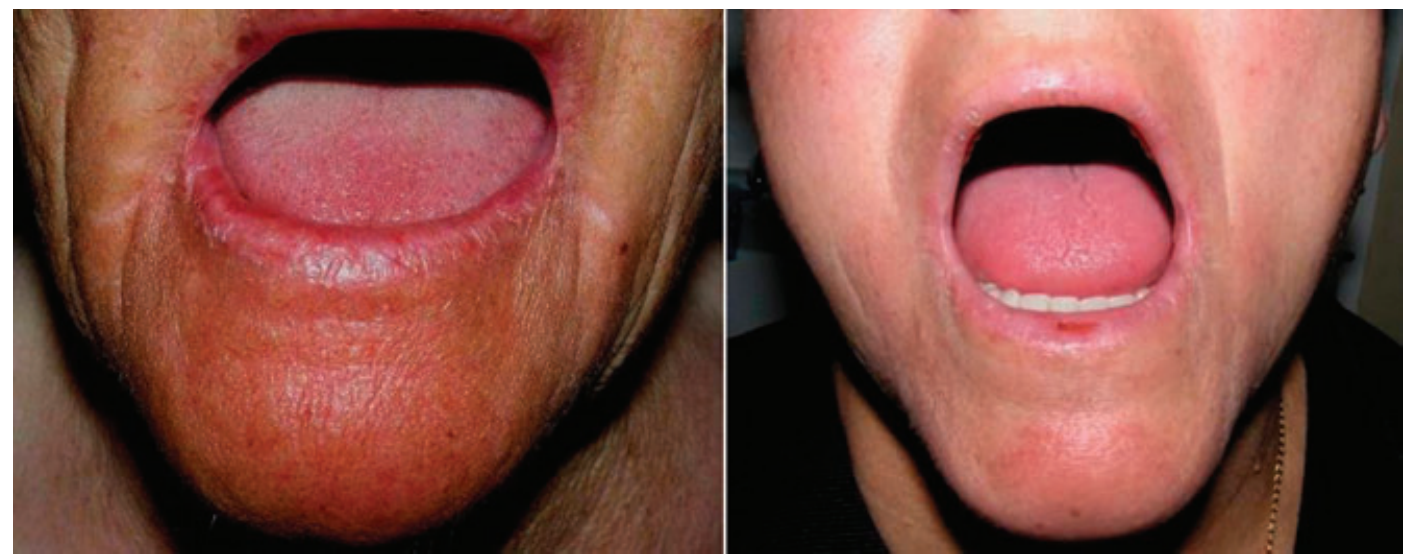

Fig. 1. Subacute pruriginous cheilitis, with erythema and scaling on the lower lip and erosions in four patients.

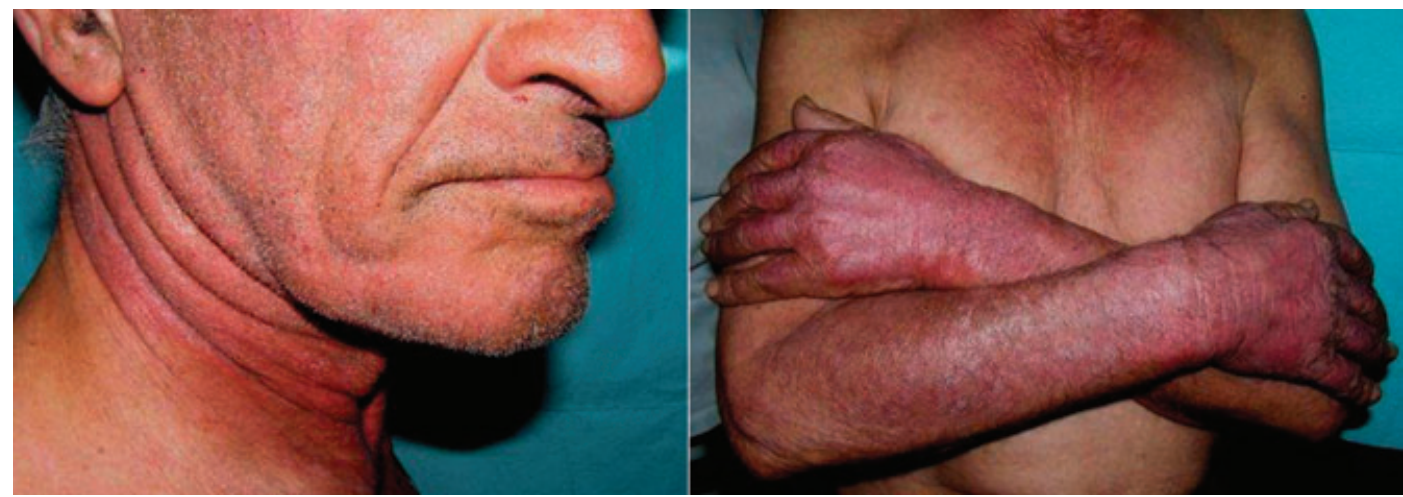

Fig. 2. Lichenified eczema of the face, neck and the ' $\mathrm{V}$ ' area of the upper chest, forearms, and dorsum of the hands in one patient.

that included sunscreens, salicylanilides and other disinfectants, phenothiazines, and NSAIDs including benzydamine (1 and 5\% petrolatum (pet.) from Bial-Aristegui $\left.{ }^{\circledR}\right)$. In suspicious cases, patients were also tested with the drug 'as is'. Allergens were applied in duplicate and irradiation of one set was performed on D2 with $5 \mathrm{~J} / \mathrm{cm}^{2}$ of UVA (Waldman ${ }^{\circledR}$ $7001 \mathrm{~K})$. Readings were performed at D2, within 30 min after irradiation and at D4, according to ICDRG guidelines.

\section{Results}

A total of 10 patients (six females/four males), aged between 21 and 84 (mean age 64.9 years) were diagnosed as photoallergic contact dermatitis from benzydamine. In nine patients there was a subacute, pruriginous cheilitis, with erythema and scaling on the lower lip in all patients and superficial erosions in four patients (Fig. 1). Eczema extended to the chin in seven patients, to the malar regions in three and was associated with erythema or eczema on the dorsum of the hands and forearms in two patients. Lesions were recurrent and, for two patients, had been occurring for more than 1 year. For 6 months, one patient had a severe lichenified eczema of the face, neck and the ' $\mathrm{V}$ ' area of the upper chest, forearms, and dorsum of the hands (Fig. 2).

All patients showed positive photopatch tests $(+$ to +++$)$ to benzydamine tested at 1 and $5 \%$ and to benzydamine containing drugs $(+$ or +++$)$ (Table 1). No reactions were observed on nonirradiated area to these allergens (Fig. 3). Additional positive reactions were observed to fragrance mix I and Myroxylon pereirae (one patient) and nickel (three patients) (Table 1).

In the nine patients who had cheilitis, their histories showed long-term regular gargling with an oral antiseptic solution containing $1.5 \%$ benzydamine (Tantum verde ${ }^{\circledR}$, Lepori Laboratories, Lisbon, Portugal). Six of these patients had oral prostheses. Two younger patients used the solution after the fitting of an orthodontic device and their cheilitis began within 2 months. An older patient with the most extensive lesions had regularly applied a topical gel containing 3\% benzydamine (Momen ${ }^{\circledR}$ gel, Lepori Laboratories, Lisbon, Portugal) to his wife's back, for about 8 months. Two patients had been on photoactive systemic drugs (amiodarone and hydrochlorothiazide) for more than 2 years and they 
Table 1. Clinical data and photopatch test results

\begin{tabular}{cllccccc}
\hline & & \multicolumn{5}{c}{ Photopatch test results } \\
\cline { 4 - 6 } Patient & Sex age & $\begin{array}{l}\text { Localization of } \\
\text { dermatitis }\end{array}$ & $\begin{array}{c}\text { Benzydamine } \\
\text { 1\% pet. }\end{array}$ & $\begin{array}{c}\text { Benzydamine } \\
5 \% \text { pet. }\end{array}$ & $\begin{array}{c}\text { Tantum } \\
\text { verde }^{\circledR}\end{array}$ & $\begin{array}{c}\text { Momen } \\
\text { gel }{ }^{\circledR}\end{array}$ & Other patch test results \\
\hline \multirow{2}{*}{1} & F 84 & L, C & NT & + & + & NT & Myroxylon pereriae, fragrance mix 1 \\
2 & M 77 & L, C, M, H, F & NT & ++ & ++ & NT & - \\
3 & F 63 & L, C, H, F & NT & ++ & ++ & ++ & Nickel \\
4 & M 71 & L, C, M & NT & ++ & ++ & NT & - \\
5 & F 21 & L & ++ & ++ & ++ & NT & - \\
6 & F 57 & L, C & ++ & ++ & ++ & ++ & - \\
7 & M 81 & LE & NT & +++ & NT & NT & Nickel \\
8 & F 72 & L, C & ++ & ++ & ++ & +++ & - \\
9 & M 74 & L & NT & ++ & ++ & NT & - \\
10 & F 49 & L, C, M & NT & ++ & ++ & ND & Nickel \\
\hline
\end{tabular}

C, chin; F, forearms; H, hands; LE, lichenified eczema on photo-exposed areas; L, lower lip; M, malar region; NT, not tested.
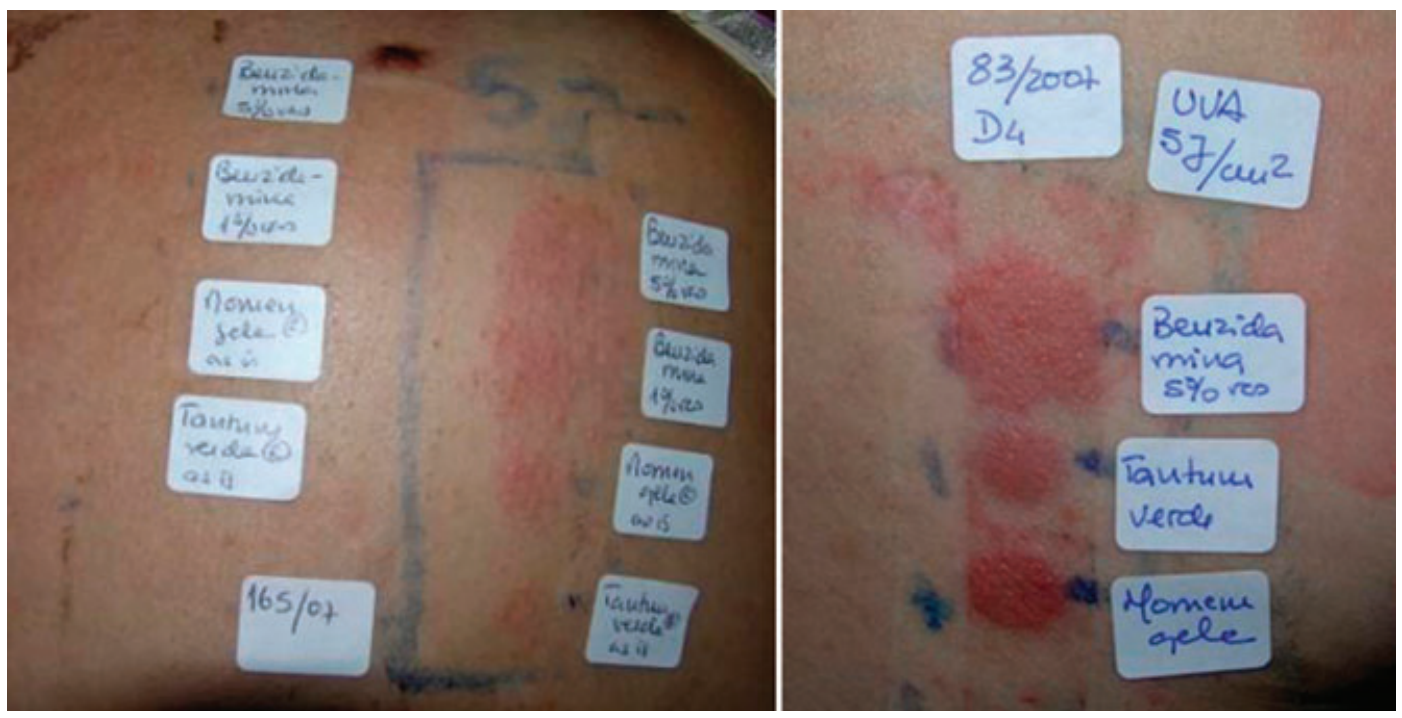

Fig. 3. Positive photopatch tests to benzydamine, Tantum verde ${ }^{\circledR}$ and Momen ${ }^{\circledR}$ gel, with negative results on non-irradiated area.

continued with no change in their doses. Lesions disappeared within a few days/weeks after benzydamine discontinuation, with no case of persistent photosensitivity. Therefore, all the reactions were considered relevant. Also, none of the other 64 patients photopatch tested with benzydamine had a positive result.

\section{Discussion}

Benzydamine has been shown to have phototoxic properties in vitro and to cause both phototoxicity and photoallergy (8). In this study, positivity of the photopatch test with benzydamine at $1 \%$ pet., the absence of reactions in the other 64 patients who were photopatch tested at 5\% pet. during this period, suggest these reactions are mainly photoallergic. Also, they were all considered relevant, with rapid improvement on drug discontinuation. The use of the gargling solution may explain the main localization on the lower lip and chin.
Although benzydamine is widely used $(1,3,7)$ and it is a known photosensitizer, with several published cases in the 1980s and 1990s (9, 10), even at our clinic (6) there are few recent reports of photoallergic contact dermatitis from benzydamine $(2,3,11)$. This may be because of the unusual presentation which does not suggest photosensitivity; the great majority of our patients presented with dermatitis involving mainly the lower lip and chin. Photoallergic contact dermatitis from benzydamine has presented as hand dermatitis from using a vaginal douche (2). Therefore, UV-irradiation of the patch tests with the mouth wash products used by the patient in cases of cheilitis is not the usual practice. As a result of its widespread use as a mouth wash, we suggest benzydamine should be included in a photopatch test series for patients being investigated for cheilitis.

Under-reporting of photosensitivity from benzydamine may also occur, because this NSAID is 
not part of most photoallergen series, and without a good history of benzydamine use it may be missed. In a recent study from Spain, where benzydamine was included in a photopatch testing series, it was the second most frequent photoallergen, after ketoprofen (12) and, in a recent report from our department, benzydamine, tested only during the latter years of the study, was one of the main photoallergens, after piroxicam and promethazine (6).

Cross-reactions with other NSAIDs have been suggested in one case (7), but, even though we did not test indomethacin, none of our patients reacted to other NSAIDs tested (ketoprofen, naproxen, ibuprofen, piroxicam, tenoxicam, and diclofenac).

Percutaneous or peroral absorption of this NSAID may have caused lesions at distant sites, as observed in four of our patients. There is a possibility that connubial photoallergic contact dermatitis as described for another NSAID, namely for ketoprofen $(13,14)$, may occur.

\section{References}

1. Henschel R, Agathos M, Breit R. Photocontact dermatitis after gargling with a solution containing benzydamine. Contact Dermatitis 2002: 47: 53.

2. Elgezua O A, Gorrotxategi P E, Garcia J G, Nieto J Á, Pérez J L. Photoallergic hand eczema due to benzydamine. Eur J Dermatol 2004: 14: 69-70.

3. Motley R J, Reynolds A J. Photodermatitis from benzydamine cream. Contact Dermatitis 1988: 19: 66.

4. Perioli L, Ambrogi V, Venezia L, Giovagnoli S, Pagano C, Rossi C. Formulation studies of benzydamine mucoadhesive formulations for vaginal administration. Drug Dev Ind Pharm 2009: 31: 1-11.

5. Kazemian A, Kamian S, Aghili M, Hashemi F A, Haddad P. Benzydamine for prophylaxis of radiation-induced oral mucositis in head and neck cancers: a double-blind placebo-controlled randomized clinical trial. Eur J Cancer Care 2009: 18: $174-178$.

6. Cardoso J C, Canelas M M, Gonçalo M, Figueiredo A. Photopatch testing with an extended series of photoallergens: a 5-year study. Contact Dermatitis 2009: 60: 325-329.

7. Buján J J, Lorentzen R I, Arechavala R S. Allergic contact dermatitis from benzydamine with probable cross-reaction to indomethacin. Contact Dermatitis 1993: 28: 111-112.

8. Moore D E, Wang J. Electron-transfer mechanisms in photosensitization by the anti-inflammatory drug benzydamine. J Photochem Photobiol B 1998; 43: 175-180.

9. Frosch P J, Weickel R. Photocontact allergy caused by benzydamine (Tantum). Hautarzt 1989: 40: 771-773.

10. Vincenzi C, Cameli N, Tardio M, Piraccini B M. Contact and photocontact dermatitis due to benzydamine hydrochloride. Contact Dermatitis 1990: 23: 25-26.

11. Giménez-Arnau A, Gilaberte M, Conde D, Espona M, Pujol R M. Combined photocontact dermatitis to benzydamine hydrochloride and the emulsifiers, Span 60 and Tween 60 contained in Tantum cream. Contact Dermatitis 2007: 57: 61-62.

12. Cuadra-Oyanguren J, Pérez-Ferriols A, Lecha-Carrelero M, Giménez-Arnau A M, Fernández-Redondo V, Ortiz de Frutos FJ, Silvestre-Salvador JF, Serra-Baldrich E. Results and assessment of photopatch testing in Spain: towards a new standard set of photoallergens. Actas Dermosifilogr 2006: 98: 96-101.

13. Hindsén M, Isaksson M, Persson L et al. Photoallergic contact dermatitis from ketoprofen induced by drug-contaminated personal objects. J Am Acad Dermatol 2004: 50: 215-219.

14. Devleeschouwer V, Roelandts R, Garmyn M, Goossens A. Allergic and photoallergic contact dermatitis from ketoprofen: results of (photo) patch testing and follow-up of 42 patients. Contact Dermatitis 2008: 58: 159-166.

\section{Address:}

Maria Miguel Canelas

Serviço de Dermatologia

Hospitais da Universidade de Coimbra

Praceta Mota Pinto

3000-075 Coimbra

Portugal

Tel: +351239400420

Fax: +351 239400490

e-mail:mariamiguelcanelas@gmail.com 